\title{
Circuit Model for Diffuse Multipath and Electromagnetic Absorption Prediction in Rooms
}

\author{
Aliou Bamba*, Wout Joseph, member IEEE, Emmeric Tanghe, Günter Vermeeren and Luc Martens \\ Ghent University / iMinds - Department of Information Technology \\ Gaston Crommenlaan 8 box 201, B-9050 Ghent-Belgium \\ Fax: +3293314 899, Tel: +3293314908 \\ *Email: aliou.bamba@intec.ugent.be
}

\begin{abstract}
We present a room electromagnetics based theory which primarily models the Diffuse Multipath Components (DMC) power density with a simple circuit model, and afterwards includes the Line-Of-Sight (LOS) component to predict the total exposure in a realistic environment. Given a human absorption cross section (ACS) and its location from a transmitter (Tx), the whole-body absorption rate $\left(\mathbf{S A R}_{w b}\right)$ can be determined by the proposed circuit model for Ultra Wide Band (UWB) and Wireless Local Area Network (WLAN) systems. The $\mathbf{S A R}_{w b}$ in humans in a realistic office environment for both UWB and WLAN systems is investigated as part of application. The theory is simulated with the Advanced Design System (ADS) software, and excellent agreement between theoretical and simulated values are obtained in terms of relative errors $(<2 \%)$. The model may be very useful for $\mathbf{S A R} \mathbf{R}_{w b}$ prediction in realistic complex indoor environments.
\end{abstract}

Keywords: room electromagnetics, Diffuse Multipath Components, whole-body absorption rate, Line-Of-sight, realistic indoor environments, diffuse scattering coefficient, lambertian diffusion, Ultra Wide Band.

\section{INTRODUCTION}

$\mathrm{M}^{-1}$ OST of people nowadays spend their time in the indoor environments. Wireless Local Area Networks (WLAN) systems are continuously used in such places, and Ultra Wide Band (UWB) systems are gaining importance. The human exposure to the electromagnetic fields (EMF) in the indoor environments therefore is of main importance. To faithfully address the human exposure, the underlying propagation phenomena must be well understood and simplified for convenient reasons. The main propagation mechanisms in the indoor environments have been investigated in [1], where it turns out that most of the interactions wave-object occur between the furnishings on the floor (desk, cardboards, machines, etc...) and possibly the devices fixed on the ceiling. The specular reflections mainly occur in that region. Besides the specular reflections, diffraction of the electromagnetic waves (EMW) occurs at corners. The walls and the large surfaces clearly cause specular reflections, but due to the irregularities at the wall surfaces and inside, the impinging EMW is converted into one reflected specular component plus a certain number of scattering reflections. Physically, the scattering waves are the ones having a different angle of departure from the reflected specular wave. The multiple diffractions and scatterings of the EMW eventually lead to a diffuse field in an indoor environment.

The diffusion phenomena in a building floor was investigated in [2], [3]. [2] has shown that the diffusion model is governed by a differential equation of the first order, which solution is comprised of exponential terms. Later, [3] developed a statistically based diffusion model via the stochastic properties of the diffusion phenomena in the indoor environments. Although these models predict excellently the diffusion behavior in the indoor environments, they require several parameters which may be difficult to obtain in practice.
The propagation phenomena are very complex in a realistic indoor environment, therefore realistic assumptions are needed to determine in a simpler and efficient manner the human exposure due to the EMW in such places.

Recently [4] addressed the diffusion issue in a single room and highlighted an interesting parameter, i.e., the reverberation time, which describes completely the diffusion behavior. Actually, the reverberation time is the decay rate of the diffuse field in a completely diffuse field, and [4] shows that the reverberation time is only dependent on the room dimensions and the surfaces (walls, ceiling, floor, ...) absorption properties. The related theory has been named by the authors room electromagnetics. Although some specular components may exist in an indoor environment, the room electromagnetics theory focuses on the diffusion effect, assuming only one specular component, typically the Line-Of-Sight (LOS) component - when it exists - in the channel response, the remainder is assumed to be part of the diffuse multipath components (DMC). This assumption certainly occurs in realistic environments since it has been shown - through measurements - that the DMC power density may represent $95 \%$ of the total power involved in a realistic indoor environment [5], [6].

This paper aims to introduce a new electrical circuit model based on the room electromagnetics theory [4], [7]. The principle of the model is shown in Fig. 1 where a room and a transmitter characteristics plus a person location are given as inputs, and the exposure in terms of the power density, hence the $\mathrm{SAR}_{w b}$ is given as the output. The transmitter characteristics $\left(\mathrm{P}_{0}, \Delta\right)$ and the person separation

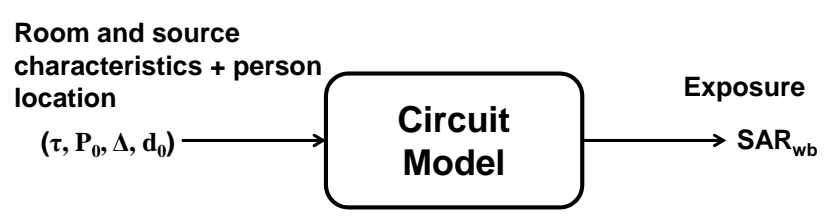

Fig. 1. Principle of the circuit model

$\left(d_{0}\right)$ from the transmitter are supposed to be known. Given a room dimensions, a simpler method to determine the reverberation time in a realistic environment without the need of carrying out any measurements or simulations is presented. The theory also applies to the adjacent rooms case and we showed an application of the circuit model in a realistic office environment. The strength of the method lies in its simplicity because it does not require any simulation of the entire room in a software, which would be very time and memory consuming - as far as the room dimensions increase - because of the difficulties of modeling the roughness of the surfaces. The novelty of the proposed method relies $i$ ) in the use 
of a room dimensions to determine the exposure of a person located inside and ii) the translation of the room electromagnetics theory into a circuit model enabling fast assessment of indoor propagation and human absorption rate. The paper is organized as follows: the model as well as the main concepts in analogy with the room electromagnetics theory are highlighted in Section II. The proposed circuit model elements are also determined. In Section III, the $\mathrm{SAR}_{w b}$ is obtained using the exposure calculation from the circuit model. An application of the theory in a realistic office environment is given in Section IV. Furthermore, the theory is simulated through electronic circuit simulating software. Finally, conclusions are drawn in Section V.

\section{MODEL}

\section{A. Overview of room electromagnetics}

Basically the room electromagnetics theory addresses the propagation in the indoor environments and considers only two propagation components: the LOS component and the DMC, as shown in Fig. 1 of [7]. Although there may exist some specular reflections in the environment, the room electromagnetics considers them as part of the DMC. The theory was introduced in [4] in analogy with the well established theory of room acoustics [8].

Let $\mathrm{P}(\mathrm{t})$ be the transmitted pulse in a room. The room electromagnetics theory states that $\mathrm{P}(\mathrm{t})$ is balanced by the decrease of the energy per second in the room and by the increase of the losses at the walls as follows [4]:

$$
P(t)=V \frac{d W}{d t}+\frac{c_{0} \eta A}{4} W
$$

where $\mathrm{V}, \mathrm{W}, \mathrm{c}_{0}, \eta$ and $\mathrm{A}$ are the room volume, the remaining energy density in the room, the light velocity in the free space, the fraction of energy absorbed by the surfaces (walls, floor, ceiling, etc.), and the total area absorbing electromagnetic radiation, respectively. The general solution of (1) is a convolution integral:

$$
W_{d}(t)=\frac{1}{V} \int_{0}^{\infty} P\left(t-t^{\prime}\right) e^{-\frac{t^{\prime}}{\tau}} d t^{\prime}
$$

where $\mathrm{W}_{d}(\mathrm{t})$ is the energy density in the room in Watt.s $/ \mathrm{m}^{3}$ and $\tau$ is the reverberation time of the room. The reverberation time depends only on the room characteristic, actually it is the decay rate of the DMC power in the room and is defined as follows [4]:

$$
\tau=\frac{4 V}{c_{0} \eta A}
$$

In a complete diffuse field, the power density is direction independent - rays come from all the directions - the energy density and the power density are therefore related by:

$$
W_{d}(t)=4 \pi \frac{I}{c_{0}}
$$

where I is the power density in Watt $/ \mathrm{m}^{2}$.

We will now distinguish between two cases:

1) the active duration of the transmitted signal $(\Delta)$ is much smaller than the reverberation time of the room, which corresponds to the UWB system case $(\Delta<<\tau)$

2) the active duration of the transmitted signal is larger than the reverberation time $(\Delta>>\tau)$, which corresponds to the WLAN systems case.

The active duration $\Delta$ is the time period during which the transmitted pulse is different from zero, i.e., $\mathrm{P}(\mathrm{t})=\mathrm{P}_{0}$. The energy evolution of the two systems is different and is shown in Fig. 2. From 0 to $\Delta_{U W B}$ (as long as the source is on) the energy of UWB systems evolves

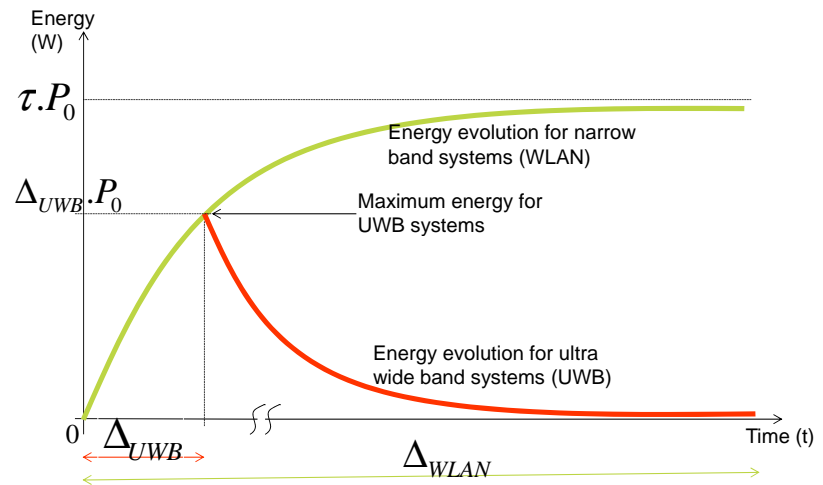

Fig. 2. Energy evolution for ultra-wide-band (UWB) and narrow-band (WLAN) systems in room electromagnetics

up to a maximum value, i.e., $\Delta P_{0}$. As soon as the source is off $\left(t>\Delta_{U W B}\right)$ the energy decreases exponentially from its peak value towards 0 with the reverberation time being the damping factor. The energy density for a small pulse $\Delta$ (UWB systems) in a room is obtained from (2):

$$
W_{d}(t)=P_{0} \frac{\tau}{V}\left(e^{\frac{\Delta}{\tau}}-1\right) e^{-\frac{t}{\tau}}
$$

The resulting total power density from $t_{0}$ in a complete diffuse field is expressed as follows [7]:

$$
I_{d}\left(t_{0}\right)=\frac{P_{0}}{\pi \eta A} e^{-\frac{t_{0}}{\tau}}
$$

where $\mathrm{P}_{0}$ is the transmitted signal.

Regarding WLAN systems where $\Delta$ is much more larger, the energy grows from 0 towards a steady state, whose value is $\tau P_{0}$. Further details about the room electromagnetics theory can be found in [4], [7].

\section{B. Model for incident power density for Ultra Wide Band (UWB) systems}

In general the reverberation time in a room is of the order of a few tens of nanoseconds and the pulse duration of the UWB systems is only a few nanoseconds (even picoseconds). The present circuit model theory is developed in analogy with [7], where the diffuse power density has been investigated. It has been assumed in that study that the pulse duration is much lower than the reverberation time. The theory developed in this part is therefore only valid for the UWB systems.

1) Model for the incident power density in the same room: Let us establish the equation governing the voltages in Fig. 3.

$$
E(t)=U(t)+R C \frac{d U(t)}{d t}
$$

where $\mathrm{E}(\mathrm{t}), \mathrm{U}(\mathrm{t}), \mathrm{R}$ and $\mathrm{C}$ are the source voltage, the voltage over the capacity, the resistor, and the capacity. This differential equation describes the charge (resp. discharge) of the capacitance when the source is on (resp. off).

The solution of (7) is given by:

$$
U(t)=\frac{1}{R C} \int_{0}^{\infty} E\left(t-t^{\prime}\right) e^{-\frac{t^{\prime}}{R C}} d t^{\prime}
$$

As the pulse signal is transmitted in the room during a period time $\Delta$, we also assume that the circuit model is fed with $\mathrm{E}(\mathrm{t})$ during the same $\Delta$, and is defined as follows:

$$
E(t)=\left\{\begin{array}{cc}
E & \text { if } \quad 0 \leq t \leq \Delta \\
0 & \text { otherwise }
\end{array}\right.
$$




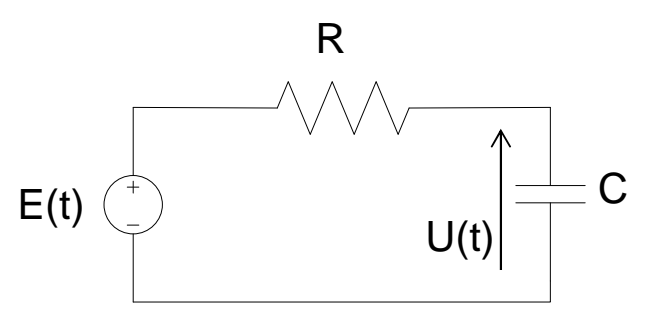

Fig. 3. Circuit model equivalent to room electromagnetics theory

Taking the $\Delta<<\tau$ assumption (UWB systems) into account, equation (8) leads then to:

$$
\begin{aligned}
U(t) & =E\left(e^{\frac{\Delta}{R C}}-1\right) e^{-\frac{t}{R C}}, \quad \mathrm{t} \geq \Delta \\
& =U_{0} e^{-\frac{t}{R C}}, \quad \mathrm{t} \geq \Delta
\end{aligned}
$$

$\mathrm{U}(\mathrm{t})$ looks like the classical expression of the voltage discharge in a capacitance $\mathrm{C}$ with its initial voltage being $\mathrm{U}_{0}$.

$$
U_{0}=E\left(e^{\frac{\Delta}{R C}}-1\right)
$$

Actually, $\mathrm{U}_{0}$ is the voltage in the capacitance just after turning the source $\mathrm{E}(\mathrm{t})$ off. Assume that the total energy in the room can be stored in the capacitance $\mathrm{C}$, the energy density (in Watt.s $/ \mathrm{m}^{3}$ ) in the room can be expressed as a function of the room volume and the capacitance energy as follows:

$$
\begin{aligned}
W_{d}(t) & =\frac{1}{2} C U^{2}(t) \times \frac{1}{V}, \quad \mathrm{t} \geq \Delta \\
& =\frac{C}{2 V} U_{0}^{2} e^{-\frac{2 t}{R C}}, \quad \mathrm{t} \geq \Delta
\end{aligned}
$$

By comparing the energy density in the circuit model i.e., (10) and in the room electromagnetics theory i.e., (5) and assuming the same decay rate, the following relations are derived:

$$
\begin{aligned}
\frac{R C}{2} & =\tau \\
R & =\frac{U_{0}^{2}}{P_{0}\left(e^{\frac{\Delta}{\tau}}-1\right)}
\end{aligned}
$$

The dimension of $\mathrm{R}$ is correct: $\frac{V^{2}}{\text { Watt }}=\Omega(\mathrm{Ohm})$. By choosing a value of $U_{0}$ and knowing $P_{0}, \Delta$ and $\tau$, the value of $\mathrm{R}$ can be easily computed. It is further demonstrated that the power density in the circuit is independent of its input voltage, justifying thereby any choice of $U_{0}$, and hence $E(t)$. Once $R$ is calculated, we use (11) to determine the value of the capacitance:

$$
C=2 \tau \frac{P_{0}\left(e^{\frac{\Delta}{\tau}}-1\right)}{U_{0}^{2}}
$$

The dimension of $\mathrm{C}$ is also correct: $\frac{s}{\Omega}=F$ (Farad).

The total power density $\mathrm{I}_{D}\left(\mathrm{~d}_{0}\right)$ at a location in the room is now calculated.

Assuming a lambertian scattering of the EMW on the surfaces - the EMW are scattered in all the directions independently on its arrival angle - as in [9], the scattering process ends up in a complete diffuse fields. The power density intensity being direction-independent in a complete diffuse field, we use (4) to determine the power density
$\mathrm{I}(\mathrm{t})$ in the capacitance as follows:

$$
\begin{aligned}
I(t) & =\frac{c_{0}}{4 \pi} \times \frac{1}{2 V} C U^{2}(t) \\
& =\frac{c_{0}}{8 \pi V} C U_{0}^{2} e^{-\frac{2 t}{R C}}
\end{aligned}
$$

The total power density $I_{D}$ from $t_{0}$ on is the total energy density from $\mathrm{t}_{0}$ divided by the time duration:

$$
I_{D}\left(t_{0}\right)=\frac{1}{\Delta} \int_{t_{0}}^{\infty} I(t) d t
$$

where $t_{0}$ is the arrival delay of the first DMC.

Taking (11) into account, a solution of (12) is:

$$
I_{D}\left(t_{0}\right)=\frac{\tau c_{0} C}{8 \pi \Delta V} U_{0}^{2} e^{-\frac{t_{0}}{\tau}}
$$

The total power density can be rewritten by:

$$
\begin{aligned}
I_{D}\left(t_{0}\right) & =\frac{\tau c_{0} C}{8 \pi \Delta V} U^{2}\left(t_{0}\right) \\
& =\frac{\tau^{2} c_{0} P_{0}}{4 \pi \Delta V}\left(e^{\frac{\Delta}{\tau}}-1\right) e^{-\frac{t_{0}}{\tau}}
\end{aligned}
$$

Note that in (13) the power density is expressed as a function of the voltage over the capacity. Moreover, (14) proves that the power density $\mathrm{I}_{D}$ in the circuit model is independent of the voltage $U_{0}$, hence $\mathrm{E}(\mathrm{t})$. It actually depends on the room characteristics ( $\mathrm{V}$ and $\tau)$ and on the source characteristics $\left(P_{0}\right.$ and $\left.\Delta\right)$.

The diffuse power density has been earlier addressed in [7] assuming that the time period $\Delta$ is much smaller compared to the reverberation time $\tau$. Since the same assumption is made in this paper, let us now calculate the power density expression in the circuit model when $\frac{\Delta}{\tau} \longrightarrow 0$ (i.e., $\Delta<<\tau$ ) for comparison purpose.

$$
\begin{aligned}
\lim _{\frac{\Delta}{\tau} \rightarrow 0} I_{D}\left(t_{0}\right) & =\lim _{\frac{\Delta}{\tau} \rightarrow 0} \frac{\tau^{2} c_{0} P_{0}}{4 \pi \Delta V}\left(e^{\frac{\Delta}{\tau}}-1\right) e^{-\frac{t_{0}}{\tau}} \\
& =\frac{\tau c_{0} P_{0}}{4 \pi V} e^{-\frac{t_{0}}{\tau}} \\
& =\frac{P_{0}}{\pi \eta A} e^{-\frac{t_{0}}{\tau}}
\end{aligned}
$$

which is the same expression as in (6).

The distance from a transmitter can be easily measured rather than the arrival time of an EMW, the power density is therefore expressed as a function of the distance. For this purpose, let us assume that the first DMC has the same arrival time as the LOS component. This hypothesis is inforced by the fact that the room electromagnetics theory assumes that the power delay profile (PDP) is comprised of only the LOS component, the remainder being the DMC (meaning that the DMC starts just after the LOS component), see Fig. 1 of [7]. Given a certain separation $d_{0}$ from the transmitter, the total power density in the circuit model is:

$$
I_{D}\left(d_{0}\right)=\frac{\tau^{2} c_{0} P_{0}}{4 \pi \Delta V}\left(e^{\frac{\Delta}{\tau}}-1\right) e^{-\frac{d_{0}}{c_{0} \tau}}
$$

2) Theory extended to adjacent rooms: The room electromagnetics theory applied to an adjacent room is addressed in [10]. The main ideas are recalled below.

Let a receiver be located in a room $\mathrm{R}_{R x}$ and a transmitter be located in the adjacent room $\mathrm{R}_{T x} . \mathrm{P}(\mathrm{t})=\mathrm{P}_{0}$ is the transmitted power in the adjacent room, and $\alpha^{2}$ is the transmission factor of the wall separating both rooms. Complete diffuse fields are assumed in both rooms in such a way that the power density is direction-independent, i.e., $I(\theta, \phi)=I$. The room $\mathrm{R}_{R x}$ is fed by two sources: 
- $\mathrm{P}_{r}(\mathrm{t})$ : which is the received LOS power from the room $\mathrm{R}_{T x}$. In fact, $\mathrm{P}_{r}$ is the transmitted LOS power $\mathrm{P}(\mathrm{t})$ scaled with the wall transmission factor and corrected with the losses due to the free space loss from the transmitter to the wall separating both rooms.

- $\mathrm{P}_{\text {exch }}$ : which is the power induced by the diffuse energy exchanged from room $\mathrm{R}_{T x}$ to room $\mathrm{R}_{R x}$ through the surface $\mathrm{S}$ between the rooms.

The received powers in the room $\mathrm{R}_{R x}$ are balanced as follows:

$$
P_{r}+P_{e x c h}=V_{R x} \frac{d W_{R x}}{d t}+\frac{c_{0} A_{R x}^{\prime}}{4} W_{R x}
$$

where $\mathrm{V}_{R x}, \mathrm{~W}_{R x}$ and $\mathrm{A}_{R x}^{\prime}$ are the volume of room $\mathrm{R}_{R x}$ (in $\mathrm{m}^{3}$ ), the energy density in the room $\mathrm{R}_{R x}$ (Watt.s $/ \mathrm{m}^{3}$ ) and the effective absorbing area in the room $\left(\mathrm{m}^{2}\right)$.

The power exchanged from the room $\mathrm{R}_{T x}$ to the room $\mathrm{R}_{R x}$ is related to the energy density in the room $\mathrm{R}_{R x}[10]$ :

$$
P_{\text {exch }}=\frac{c_{0} S}{4} W_{R x}
$$

The energy density is a solution of (17), and is given by:

$$
W_{R x}(t)=\frac{1}{V_{R x}} \int_{0}^{\infty} P_{r}\left(t-t^{\prime}\right) e^{-\frac{t^{\prime}}{\tau_{a}}} d t^{\prime}
$$

where $\tau_{a}$ is the reverberation time in the room $\mathbf{R}_{R x}$ when the transmitter is located in the room $\mathrm{R}_{T x}$, and $\mathrm{P}_{r}(\mathrm{t})$ is defined as:

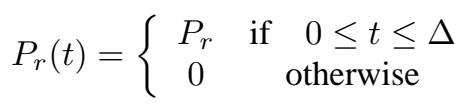

Assume that the shortest distance from the transmitter to the wall separating both rooms is $d_{T W}$, and that a LOS plane wave impinges on the wall $\mathrm{S}$, the received LOS power in room $\mathrm{R}_{R x}$ is:

$$
P_{r}=\alpha^{2} \frac{P_{0}}{4 \pi d_{T W}^{2}} S
$$

The energy density in the room $\mathrm{R}_{R x}$ is then (solution of (18)):

$$
\begin{aligned}
W_{R x}(t) & =\frac{P_{r} \tau_{a}}{V_{R x}}\left(e^{\frac{\Delta}{\tau_{a}}}-1\right) e^{-\frac{t}{\tau_{a}}} \\
& =\frac{\tau_{a}}{V_{R x}} \frac{\alpha^{2} P_{0} S}{4 \pi d_{T W}^{2}}\left(e^{\frac{\Delta}{\tau_{a}}}-1\right) e^{-\frac{t}{\tau_{a}}}
\end{aligned}
$$

The reverberation times $\tau$ (transceivers in the same room) and $\tau_{a}$ (transceivers in adjacent rooms) are linked via the following formula [10]:

$$
\begin{aligned}
\tau_{a} & =\frac{\tau}{1-\frac{S}{A_{R x}^{\prime}}} \\
& =\frac{\tau}{1-\frac{S c_{0} \tau}{4 V_{R x}}}
\end{aligned}
$$

In analogy to the theory developed for the same room, the circuit model for adjacent rooms is shown in Fig. 4. The resistance is $\mathrm{R}_{a}$ and the capacitance is $\mathrm{C}_{a}$. Thus, comparing the energy density in the circuit model (10) and in the room electromagnetics theory (19), we derived the following relations:

$$
\begin{aligned}
\frac{R_{a} C_{a}}{2} & =\tau_{a} \\
R_{a} & =\frac{4 \pi d_{T W}^{2}}{\alpha^{2} S} \times \frac{U_{0}^{2}}{P_{0}\left(e^{\frac{\Delta}{\tau_{a}}}-1\right)}
\end{aligned}
$$

Since $\mathrm{R}_{a}$ is computed from (21), the capacitance $\mathrm{C}_{a}$ is computed from (20) with the knowledge of $\tau_{a}$. Likewise to the single room

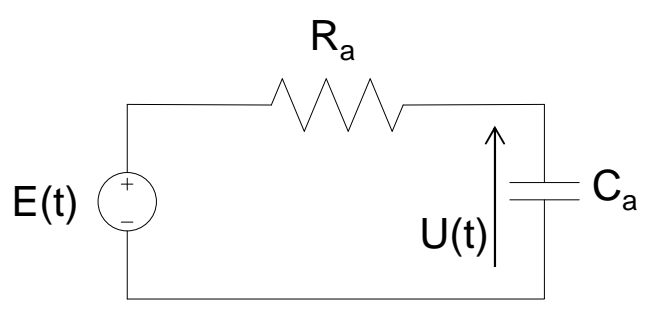

Fig. 4. Circuit model equivalent to room electromagnetics theory for adjacent rooms

case, the power density expression for adjacent rooms is finally:

$$
\begin{aligned}
I_{D, a}\left(t_{0}\right) & =\frac{\tau_{a} c_{0} C_{a}}{8 \pi \Delta V_{R x}} U^{2}\left(t_{0}\right) \\
& =\frac{\tau_{a}^{2} c_{0} P_{r}}{4 \pi \Delta V_{R x}}\left(e^{\frac{\Delta}{\tau_{a}}}-1\right) e^{-\frac{t_{0}}{\tau_{a}}} \\
I_{D, a}\left(d_{0}\right) & =\frac{\tau_{a}^{2} c_{0}}{4 \pi \Delta V_{R x}} \frac{\alpha^{2} P_{0} S}{4 \pi d_{T W}^{2}}\left(e^{\frac{\Delta}{\tau_{a}}}-1\right) e^{-\frac{d_{0}}{c_{0} \tau_{a}}}
\end{aligned}
$$

3) Including the LOS component in the model: The goal of the circuit model is primarily to model the power density of the DMC. However, in order to use this circuit model to address the human exposure, it should include the LOS component power density as well since the $\mathrm{SAR}_{w b}$ is induced by both sources. Note that the human exposure is expressed in terms of the SAR in the radio frequency region [11]. Given an incident LOS plane wave, its power density at a certain distance $\mathrm{d}_{0}$ is:

$$
I_{L}=\frac{\alpha^{2} P_{0}}{4 \pi d_{0}^{2}}
$$

where $\mathrm{I}_{L}, \alpha^{2}, \mathrm{~d}_{0}$ are the LOS component power density (in Watt $/ \mathrm{m}^{2}$ ), the walls transmission factor (if there is any wall between Tx and $\mathrm{Rx}$ ) and the separation from the transmitter (in meter), respectively. For the same room scenario, $\alpha^{2}=1$ since there is no transmission through the wall.

Consider that the LOS power density illuminates a person with a certain $\mathrm{ACS}_{L O S}$, the power absorbed by that person is:

$$
\begin{aligned}
P_{a b s}^{L O S} & =I_{L} \times A C S_{L O S} \\
& =\frac{\alpha^{2} P_{0}}{4 \pi d_{0}^{2}} \times A C S_{L O S}
\end{aligned}
$$

Since the voltage of the circuit model has not yet been set to a particular value, the peak voltage $U_{0}$ in the capacitance can be defined as follows:

$$
\begin{aligned}
& U_{0}^{2}=Z_{0} \times P_{a b s}^{L O S} \\
& U_{0}=\sqrt{Z_{0} \times \frac{\alpha^{2} P_{0}}{4 \pi d_{0}^{2}} \times A C S_{L O S}}
\end{aligned}
$$

where $Z_{0}$ is the free-space impedance (in $\Omega$ ). Since $U_{0}$ is known, the circuit model input voltage $\mathrm{E}(\mathrm{t})$ is determined with (9).

Finally, the LOS component power density in the circuit model is given by:

$$
I_{L}=\frac{U_{0}^{2}}{Z_{0} \times A C S_{L O S}}
$$

which is only dependent on the capacitance peak voltage and the human body surface illuminated by the LOS plane wave. The circuit model derived here determines the exposure (and afterwards the $\mathrm{SAR}_{w b}$ ) for only one person at one position. If different persons stand 
at different distances from the transmitter, different circuit models are then needed. So in general, the $\mathrm{SAR}_{w b}$ in different humans in rooms will be predicted by a set of circuit models (Section IV).

[12] has shown that the highest absorption rate occurs for plane waves incident to the front of the phantom, or person. The wholebody absorption in terms of illuminated surface depends highly on the azimuth of the incident plane wave illuminating the person [13], [14], on the plane wave polarization as well. Hence, the $\operatorname{ACS}_{L O S}$ depends on the incident LOS component azimuth and polarization but this issue is considered as part of another research. The $\mathrm{ACS}_{L O S}$ is supposed to be a known parameter, which already takes the incident LOS plane wave directions and polarization into account.

\section{Model for incident power density for WLAN - narrow band -} systems $(\Delta>>\tau)$

Nowadays, the WLAN IEEE 802.11 standard systems are widely deployed in residential/office environments. For these systems, the active duration of the transmitted signal $(\Delta)$ is of the order of a few hundreds of microseconds [15] whereas the reverberation time is still about a few tens of nanoseconds. The $\Delta<<\tau$ assumption therefore does not hold anymore and this case needs to be addressed otherwise. From the room electromagnetics point of view, the energy density evolution is still governed by (2) and (18) for the same room and the adjacent rooms, respectively. The active duration of the transmitted pulse is several thousands times the reverberation time, the energy density can therefore reach the steady state as stated earlier. The energy density for the same room can be found by integrating (1) from 0 to $t$.

$$
W_{d}(t)=\frac{\tau P_{0}}{V}\left(1-e^{-\frac{t}{\tau}}\right)
$$

Similarly to the UWB case, the power density for the narrow-band systems is calculated with the $\Delta>>\tau$ assumption. Given the energy density in (25) and assuming complete diffuse fields, the power density is:

$$
\begin{aligned}
I(t) & =\frac{c_{0}}{4 \pi} W_{d}(t) \\
& =\frac{c_{0}}{4 \pi V} \tau P_{0}\left(1-e^{-\frac{t}{\tau}}\right)
\end{aligned}
$$

The signal is transmitted during $\Delta$, the total power density from $\mathrm{t}_{0}$ on is then:

$$
\begin{aligned}
I_{D}\left(t_{0}\right) & =\frac{1}{\Delta} \int_{t_{0}}^{\Delta} I(t) d t, \quad \mathrm{t}_{0}<\Delta \\
& =\frac{c_{0}}{4 \pi V} \tau P_{0}\left(1-\frac{t_{0}}{\Delta}+\frac{\tau}{\Delta} e^{-\frac{\Delta}{\tau}}-\frac{\tau}{\Delta} e^{-\frac{t_{0}}{\tau}}\right)
\end{aligned}
$$

Because of the narrow-band assumption $(\Delta>>\tau)$, the two last terms of (26) tend towards 0 , so they are negligible. Considering a residential or an office environment with a maximum length of $300 \mathrm{~m}$ leads to a maximum arrival delay $\mathrm{t}_{0}$ of about $1000 \mathrm{~ns} . \Delta$ is of order of a few hundred of microseconds, the term $\frac{t_{0}}{\Delta}$ can thereby be neglected, leading to the final expression of the average power density:

$$
\begin{aligned}
I_{D}\left(t_{0}\right) & =\frac{c_{0}}{4 \pi V} \tau P_{0} \\
& =\frac{P_{0}}{\pi \eta A}
\end{aligned}
$$

Unlike to the UWB case, the total power density for the narrow band system is independent on the distance from the transmitter $\left(\mathrm{d}_{0}\right)$; it rather depends on the room and Tx characteristics. Since the total power density exhibits no exponential decay, it is not modeled with a circuit model.

Likewise, the energy density for the adjacent rooms case is found by integrating (18). Following the same procedure, the total power density is as follows:

$$
\begin{aligned}
I_{D, a}\left(t_{0}\right) & =\frac{c_{0}}{4 \pi V_{R x}} \tau_{a} P_{r} \\
& =\frac{c_{0} \tau_{a}}{4 \pi V_{R x}} \times \frac{\alpha^{2} P_{0} S}{4 \pi d_{T W}^{2}}
\end{aligned}
$$

\section{Determination of THE SAR S $_{b}$ From the EXPOSURE}

\section{A. Expression for ultra-wide-band systems}

The power densities for both sources (DMC and LOS components) are known via the circuit model, the $\mathrm{SAR}_{w b}$ is then easily derived:

$$
\begin{aligned}
S A R_{w b} & =S A R_{D M C}+S A R_{L O S} \\
& =\frac{1}{m}\left(I_{D} \times A C S+I_{L} \times A C S_{L O S}\right) \\
& =\frac{1}{m}\left(\frac{\tau c_{0} C}{8 \pi \Delta V} U^{2}\left(\frac{d_{0}}{c_{0}}\right) A C S+\frac{U_{0}^{2}}{Z_{0}}\right)
\end{aligned}
$$

where $\mathrm{SAR}_{w b}$ and $\mathrm{m}$ are the whole-body specific absorption rate in Watt $/ \mathrm{kg}$ and the mass of the person in $\mathrm{kg}$. For the adjacent rooms case, $\tau$ and $\mathrm{C}$ have to be replaced by $\tau_{a}$ and $\mathrm{C}_{a}$, respectively.

\section{B. Expression for narrow-band systems}

The $\mathrm{SAR}_{w b}$ is derived in a similar way as for the wide-band case. The expression is as follows:

$$
\begin{aligned}
S A R_{w b} & =S A R_{D M C}+S A R_{L O S} \\
& =\frac{1}{m}\left(I_{D} \times A C S+I_{L} \times A C S_{L O S}\right)
\end{aligned}
$$

The $\mathrm{SAR}_{w b}$ in the same and adjacent rooms are then given by the following:

$$
S A R_{w b}\left\{\begin{array}{l}
=\frac{1}{m}\left(\frac{c_{0} \tau P_{0}}{4 \pi V} A C S+\frac{P_{0}}{4 \pi d_{0}^{2}} A C S_{L O S}\right) \\
\text { for the same room } \\
=\frac{1}{m}\left(\frac{c_{0} \tau_{a}}{4 \pi V} \frac{\alpha^{2} P_{0} S}{4 \pi d_{T W}^{2}} A C S+\frac{\alpha^{2} P_{0}}{4 \pi d_{0}^{2}} A C S_{L O S}\right) \\
\text { for the adjacent rooms }
\end{array}\right.
$$

where $\mathrm{d}_{T W}$ and $\mathrm{d}_{0}$ are the shortest distance between the transmitter and the adjacent room, and the distance separating the transmitter to the person, respectively.

\section{APPLICATION}

In the following section, we show how the circuit model can be used in practice in a realistic environment.

\section{A. Determination of the reverberation time}

Depending on the trade-off between the accuracy and the simplicity, the reverberation time can be numerically determined using the radiosity method [16], or by carrying out measurements [10], respectively. Our method aims to avoid the need of performing measurements or computations, which are both complicated and time consuming. The reverberation time is rather determined in a simpler but practical way, using the effective roughness of the surfaces and a lambertian diffusion model as in [9]. The effective roughness assume not only the roughness on the surfaces, but also inside them. The well established reverberation time expression in [4] is used to compute the reverberation time of the room $i$ as follows:

$$
\tau_{i}=\frac{4 V_{i}}{c_{0} \eta A_{i}}
$$


where $\tau_{i}, \mathrm{~V}_{i}, \eta$, and $\mathrm{A}_{i}$ are the reverberation time, the volume of the room, the fraction of energy absorbed by the areas in the room, and the total area taking part in the diffusion, respectively.

The main challenge resides in the determination of the fraction of energy $\eta$ absorbed by the areas (the walls, the floor and the ceiling). Let us suppose the following assumptions:

- $\eta$ is supposed to be uniform for all the absorbing areas (walls, ceiling, floor), i.e., it can be seen as an average $\eta$. In the diffuse scattering literature $(1-\eta)$ is assimilated to the diffuse scattering coefficient, which is the ratio of the incident wave being scattering.

- the surfaces of the walls, the ceiling, and the floor are assumed to be Lambertian (i.e., the pattern of the scattered rays is independent of the incident EMW direction) and perfectly diffuse.

The propagation phenomena resulting from the interaction of an incident plane wave and a rough surface are assumed to be lambertian scattering. The Lambert's law approximates well the reflectance of some surfaces such as the flat paints [17], hence the law is quite suitable for the indoor environments. According to the Lambert's law, the bidirectional reflectance of a perfectly diffuse surface is [17]:

$$
r_{L}\left(\theta_{i}\right)=\frac{1}{\pi} \cos \left(\theta_{i}\right)
$$

where $\mathrm{r}_{L}$, and $\theta_{i}$ are the bidirectional reflectance, and the angle between the incident plane wave and the normal of the surface, respectively. The reflectance is defined in [17] as the fraction of the incident light scattered diffusely into many directions by a geometrically complex medium. The term bi-directional refers to the orientation of the source (one incident wave) and the detector (one emerging wave). Actually, we are interested in the directionalhemispherical reflectance since the source is a LOS component and the diffuse waves emerge in the whole upper half-space of the surface (rays emerge from all directions in a diffuse scenario). The directional-hemispherical reflectance is called scattering coefficient elsewhere in the literature. The actual expression of the directionalhemispherical reflectance is complex because it involves the properties of the particles forming the surfaces [17] and inside, which is very difficult - if not impossible - to obtain in practice. Because of the lack of informations about these particles, the directionalhemispheral reflectance $(0 \leq \rho \leq 1)$ is determined by integrating the bidirectional reflectance over the half upper space as follows:

$$
\begin{aligned}
\rho & =\frac{1}{2 \pi} \int_{\varphi=0}^{2 \pi} \int_{\theta_{s}=0}^{\frac{\pi}{2}} r_{L}\left(\theta_{i}\right) \sin \left(\theta_{s}\right) d \theta_{s} d \varphi \\
& =r_{L}\left(\theta_{i}\right)
\end{aligned}
$$

where $\theta_{s}$ is the angle between the normal to the surface and a given scattering ray, and $\varphi$ is the azimuthal angle.

The incident source is a LOS plane wave perpendicular to the surfaces $\left(\theta_{i}=0\right)$, therefore the calculation of (32) leads to $\rho=\frac{1}{\pi}=0.318$. Note that using a directive scattering pattern instead of a lambertian model, [18] has found that a scattering coefficient of 0.4 is the best value for an office environment. Moreover, [9] used a lambertian model and found a scattering coefficient of 0.316. Regarding these values, the scattering coefficient determined here agrees with the values in the literature.

The fraction of the diffuse energy absorbed by the surface is hence:

$$
\begin{aligned}
\eta & =1-\rho \\
& =0.68
\end{aligned}
$$

However, (31) is only valid for small values of $\eta$ [16]. When dealing with higher $\eta$, it is corrected by the following [8]:

$$
\eta_{c}=-\log (1-\eta) *\left(1+\frac{\gamma^{2}}{2} \log (1-\eta)\right)
$$

where $\eta_{c}$ is the corrected $\eta$, and $\gamma^{2}$ is a parameter taking into account the relative dimensions of the room. Actually it is the relative variance of the path length distributions in a room, the corresponding values can be found in [8].

\section{B. Configuration}

The configuration of the Fig. 5 is considered. In the following sections, the $\mathrm{SAR}_{w b}$ in the persons indicated in Fig. 5 as $\mathrm{p}_{11}, \mathrm{p}_{12}$, $\mathrm{p}_{21}$ and $\mathrm{p}_{31}$ is investigated via the circuit model. The notation $\mathrm{p}_{i j}$ designates the person number $j$ in the room number $i$. A transmitter

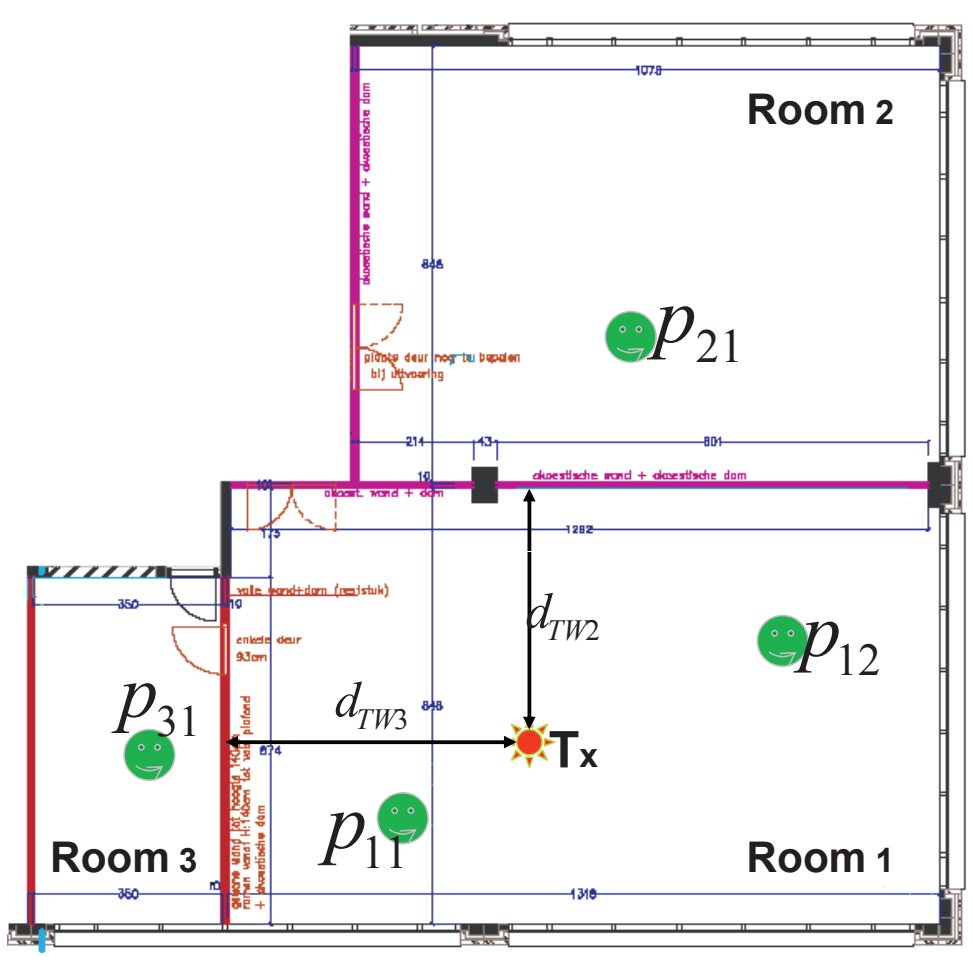

Fig. 5. Ground plan of the investigated rooms with people locations

Tx is located in the room Rooml (R1) which contains 2 persons: $\mathrm{p}_{11}$ and $\mathrm{p}_{12}$. The adjacent rooms Rooml (R2) and Room3 (R3) contain also 2 persons $\mathrm{p}_{21}$ and $\mathrm{p}_{31}$, respectively. The surface of the walls separating Room1-Room 2 and Room1-Room 3 are $\mathrm{S}_{12}=28.30 \mathrm{~m}^{2}$ and $\mathrm{S}_{13}=18.00 \mathrm{~m}^{2}$, respectively. Once more, $\mathrm{S}_{i j}$ designates the surface of the wall separating the room $i$ and the room $j$. Because the smooth glasses do not diffuse the electromagnetic waves, the glasses have not been accounted in the absorbing area computation. The transmitter is located at $\mathrm{d}_{T W 2}=4.7 \mathrm{~m}$ and $\mathrm{d}_{T W 3}=5.65 \mathrm{~m}$ from the wall $\mathrm{S}_{12}$ and $\mathrm{S}_{13}$, respectively. The distance $\mathrm{d}_{T W j}$ is the shortest distance between the transmitter and the room $R j$. Using the room dimensions and the equations (33), (31) the reverberation time of $32.06 \mathrm{~ns}, 31.33 \mathrm{~ns}$ and $20.13 \mathrm{~ns}$ are obtained for the rooms R1, R2, and R3, respectively. The rooms characteristics and the reverberation time values are summarized in Table I. The relative dimensions are defines as $\left(1: \frac{\text { width }}{\text { height }}: \frac{\text { length }}{\text { height }}\right)$, where the result of each ratio is rounded to the nearest integer between the following set of integers, i.e., 1, 2,5 and 10 defined in [8]. 


\begin{tabular}{|l|l|l|l|l|l|l|}
\hline Room & $\begin{array}{l}\text { relative } \\
\text { dimensions }\end{array}$ & $\gamma^{2}$ & $\eta_{c}$ & $\begin{array}{l}\text { Volume } \\
\left(\mathrm{m}^{3}\right)\end{array}$ & $\begin{array}{l}\text { Area } \\
\left(\mathrm{m}^{2}\right)\end{array}$ & $\begin{array}{l}\tau \\
(\mathrm{ns})\end{array}$ \\
\hline $\mathrm{R} 1$ & $1: 2: 5$ & 0.403 & 0.4455 & 300 & 280 & 32.06 \\
\hline $\mathrm{R} 2$ & $1: 2: 5$ & 0.403 & 0.4455 & 245 & 234 & 31.33 \\
\hline R3 & $1: 1: 2$ & 0.356 & 0.4513 & 63.10 & 92.60 & 20.13 \\
\hline
\end{tabular}

TABLE I

CHARACTERISTICS OF THE THREE INVESTIGATED ROOMS

\section{Determination of the circuit model elements for UWB systems}

Consider a UWB system operating at $2.5 \mathrm{GHz}$ within a bandwidth of $1 \mathrm{GHz}$. The EIRP (Effective Isotropically Radiated Power) level limitations for the UWB systems has been issued by the US frequency regulator (Federal Communications Commission). For the indoor communications, a power spectral density of about $51.30 \mathrm{dBm} / \mathrm{MHz}$ sets the transmissions level for communications between 2 and $3 \mathrm{GHz}$ [19]. A $1 \mathrm{GHz}$ bandwidth leads then to an EIRP of $7.40 \mathrm{~mW}$ and an active pulse duration $\Delta$ of $1 \mathrm{~ns}$. For each exposed person, the elements to determine are the resistance $\mathrm{R}$, the capacitance $\mathrm{C}$ and the peak voltage $\mathrm{U}_{0}$ in the capacitance. The input voltage of the circuit model is expressed as a function of the person ACS. A typical value of $0.35 \mathrm{~m}^{2}$ - based on measurements on real humans - is chosen as the $\mathrm{ACS}_{w b}$ (in diffuse field) value, which in turn leads to an $\mathrm{ACS}_{L O S}$ of $0.175 \mathrm{~m}^{2}$ for a frontal LOS plane wave illumination at $2.3 \mathrm{GHz}$ [10]. The penetration loss of the wall $(7 \mathrm{~dB})$ is accounted for the adjacent rooms elements computation. Table II lists the resulting equivalent circuit elements for the configuration of Fig. 5 and the UWB specifications.

\begin{tabular}{|l|l|l|l|l|l|}
\hline Person & $\begin{array}{l}\text { distance } \\
(\mathrm{m})\end{array}$ & $\begin{array}{l}\text { voltage } \\
\mathrm{U}_{0} \text { in } \\
(\mathrm{mV})\end{array}$ & $\begin{array}{l}\text { resistance } \\
\mathrm{R}(\Omega)\end{array}$ & $\begin{array}{l}\text { capacitance } \\
\mathrm{C}(\mathrm{nF})\end{array}$ & $\begin{array}{l}\tau \text { or } \tau_{a} \\
(\mathrm{~ns})\end{array}$ \\
\hline $\mathrm{p} 11(\mathrm{R} 1)$ & 3 & 66 & 18.58 & 3.45 & 32.06 \\
\hline $\mathrm{p} 12(\mathrm{R} 1)$ & 6 & 33 & 4.65 & 13.80 & 32.06 \\
\hline p21 (R2) & 10 & 18 & 22.82 & 3.77 & 43.00 \\
\hline p31 (R3) & 8 & 22 & 63.52 & 1.11 & 35.36 \\
\hline
\end{tabular}

TABLE II

CIRCUIT MODEL ELEMENTS

1) Whole-body SAR from theoretical formula: Equations (16) and (23) can be used to compute the diffuse power density in the room $\mathrm{R} 1$ and its adjacent rooms (R2 and R3), respectively and the LOS component power density is determined with (24). Assuming that the person mass is about $70 \mathrm{~kg}$, the theoretical $\mathrm{SAR}_{w b}$ in a person is determined and the results are shown in Table III. As expected,

\begin{tabular}{|l|l|l|l|l|}
\hline Person & $\begin{array}{l}\text { distance } \\
(\mathrm{m})\end{array}$ & $\begin{array}{l}\mathrm{I}_{D} \\
\left(\mathrm{~mW} / \mathrm{m}^{2}\right)\end{array}$ & $\begin{array}{l}\mathrm{I}_{L} \\
\left(\mathrm{~mW} / \mathrm{m}^{2}\right)\end{array}$ & $\begin{array}{l}\mathrm{SAR}_{w b} \\
(\mu \mathrm{W} / \mathrm{kg})\end{array}$ \\
\hline $\mathrm{p} 11(\mathrm{R} 1)$ & 3 & $1.4010^{-2}$ & $6.6010^{-2}$ & 0.235 \\
\hline $\mathrm{p} 12(\mathrm{R} 1)$ & 6 & $1.0310^{-2}$ & $1.6510^{-2}$ & $9.2710^{-2}$ \\
\hline $\mathrm{p} 21(\mathrm{R} 2)$ & 10 & $1.1810^{-3}$ & $4.9110^{-3}$ & $1.8210^{-2}$ \\
\hline $\mathrm{p} 31(\mathrm{R} 3)$ & 8 & $1.7010^{-3}$ & $7.3410^{-3}$ & $2.68510^{-2}$ \\
\hline
\end{tabular}

THEORETICAL WHOLE-BODY SAR FOR THE SCENARIO OF FIG. 5

the higher $\mathrm{SAR}_{w b}$ occurs in the person $\mathrm{p}_{11}$ because of its vicinity to the transmitter. The lowest $\mathrm{SAR}_{w b}$ is induced in person $\mathrm{p}_{21}$ since he is the furthest from the Tx antenna. The most relevant remark here is the fact that the diffuse fields contribution is accounted for through a circuit model - to assess the $\mathrm{SAR}_{w b}$. It turns out that the contribution of the diffuse power density $\mathrm{I}_{D}$ (for all the investigated scenarios) can not be neglected compared to the LOS power density $\mathrm{I}_{L}$.

2) Whole-body SAR from the circuit model simulation: The Advanced Design System (ADS) [20] is used to simulate the circuit model. The circuit model of Fig. 3 is used to emulate the exposure of the configuration shown in Fig. 5. Because the $\mathrm{R}$ and $\mathrm{C}$ values are now known, (9) is used to computed the actual value of the voltage $\mathrm{E}$ feeding the source for each circuit model. As mentioned in Section II-B, the source is turned off after $1 \mathrm{~ns}$ since the power is transmitted during $1 \mathrm{~ns}$ in the room R1. The circuit model of one person $\left(\mathrm{p}_{11}\right)$ is displayed in Fig. 6. The voltage in a capacitance is

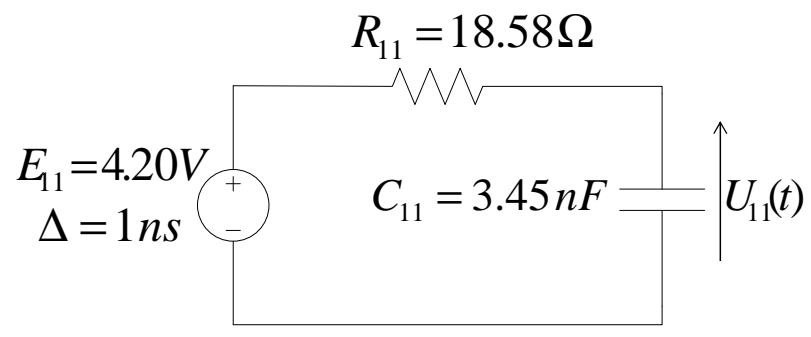

Fig. 6. Circuit model of person p11. The source has been turn off after $1 \mathrm{~ns}$. A transient simulation was used to see the voltage decay over $600 \mathrm{~ns}$ with a step of $0.1 \mathrm{~ns}$.

time dependent and frequency independent, a transient simulation is therefore used for each case. The schematic is the same for any other person of Fig. 5, unless the parameter R, C and E are replaced with the appropriate ones, whose values are in Tables II and IV. The result of the simulation in Fig. 6 is the voltage discharge in the capacitance, as shown in Fig. 7. The separation Tx-person in meter

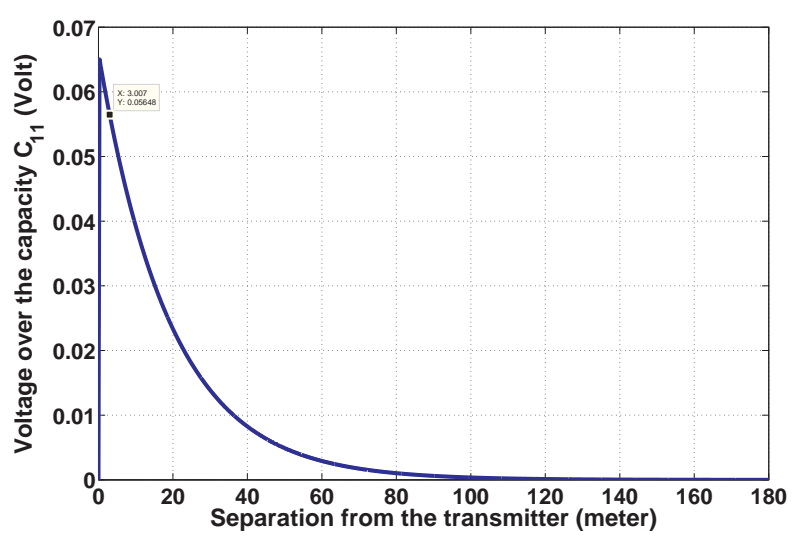

Fig. 7. Capacity voltage for person p11

is given on the $\mathrm{X}$-axis and the capacity voltage in Volt on the Yaxis. A marker on the plot shows the person p11 location and the voltage over the related capacity. It can be checked that the value of $\mathrm{U}_{0}$ in Table II corresponds to the peak value in the voltage plot of the Fig. 7. Knowing the capacity voltage at a certain time (hence at a certain distance), (29) enables the determination of the $\mathrm{SAR}_{w b}$ absorbed by a person in the room. The simulated results in terms of the $\mathrm{SAR}_{w b}$ are shown in Table IV, and agree excellently with those of Table III. Recall the mass and the $\mathrm{ACS}_{w b}$ of each person are $70 \mathrm{~kg}$ and $0.35 \mathrm{~m}^{2}$, respectively. The relative error $(\triangle \mathrm{SAR})$ is defined 
as the difference between the theoretical and the simulated values in percentage of the theoretical value. Its values are also shown in Table IV. It is worthy to mention that the simulation part does not aim to validate the theory, it rather shows a method to implement the theory in real scenarios.

The present circuit model theory $i$ ) is based on the room electromagnetics theory which has already been validated through measurements in [4] and ii) the room electromagnetics based $\mathrm{SAR}_{w b}$ assessment has already been validated through measurements and numerical simulations in [21]. The presented circuit model theory is based on these previous validations.

\begin{tabular}{|l|l|l|l|l|}
\hline Person & $\begin{array}{l}\text { distance } \\
\mathrm{d}_{0} \text { in }(\mathrm{m})\end{array}$ & $\begin{array}{l}\mathrm{E} \\
(\mathrm{V})\end{array}$ & $\begin{array}{l}\mathrm{SAR}_{w b}^{\text {sim }} \\
(\mu \mathrm{W} / \mathrm{kg})\end{array}$ & $\begin{array}{l}\Delta \mathrm{SAR} \\
(\%)\end{array}$ \\
\hline p11 (R1) & 3 & 4.20 & 0.234 & 0.42 \\
\hline p12 (R1) & 6 & 2.10 & $9.2010^{-2}$ & 0.75 \\
\hline p21 (R2) & 10 & 1.54 & $1.8010^{-2}$ & 1.1 \\
\hline p31 (R3) & 8 & 1.54 & $2.6710^{-2}$ & 0.56 \\
\hline
\end{tabular}

TABLE IV

WHOLE-BODY SAR FROM SIMULATION FOR THE SCENARIO OF FIG. 5

\section{Application to 802.11 systems}

Consider an IEEE $802.11 \mathrm{~g}$ system operating at $2.4 \mathrm{GHz}$ in the same indoor environment shown in Fig. 5. The characteristics of the system are retrieved from [15] and are the following: EIRP (Effective Isotropically Radiated Power) of $100 \mathrm{~mW}$, and an active duration of the transmitted pulse of $209 \mu \mathrm{s}(\Delta)$. The exposure of the persons for such system are listed in Table V. Equations (27), (28), and (24) have been used to compute $\mathrm{I}_{D}, \mathrm{I}_{D, a}$ and $\mathrm{I}_{L}$, respectively. It is noticed that the $\mathrm{SAR}_{w b}$ for the WLAN system (Table V) is

\begin{tabular}{|c|c|c|c|c|c|}
\hline Person & $\begin{array}{l}\text { distance } \\
\text { (m) }\end{array}$ & $\begin{array}{l}\tau \text { or } \tau_{a} \\
(\mathrm{~ns})\end{array}$ & $\begin{array}{l}\mathrm{I}_{D} \quad \text { or } \\
\mathrm{I}_{D, a} \\
\left(\mathrm{~mW} / \mathrm{m}^{2}\right)\end{array}$ & $\begin{array}{l}\mathrm{I}_{L} \\
\left(\mathrm{~mW} / \mathrm{m}^{2}\right)\end{array}$ & $\begin{array}{l}\mathrm{SAR}_{w b} \\
(\mu \mathrm{W} / \mathrm{kg})\end{array}$ \\
\hline p11 (R1) & 3 & 32.06 & 0.255 & 0.884 & 3.485 \\
\hline p12 (R1) & 6 & 32.06 & 0.255 & 0.221 & 1.80 \\
\hline p21 (R2) & 10 & 43.00 & 0.034 & 0.0637 & 0.33 \\
\hline p31 (R3) & 8 & 35.36 & 0.048 & 0.0995 & 0.49 \\
\hline
\end{tabular}

EXPOSURE TO IEEE 802.11 G SYSTEM

higher than the absorption rate for the UWB system (Table III). One may think that this is due to the higher transmitted power in the WLAN system case; this is correct if an absolute comparison of the values is made. However, let's do a relative comparison of the different powers involved in the absorption. The ratio of the LOS power densities for both systems $\frac{I_{L, U W B}}{I_{L, W L A N}}$ and the ratio of the diffuse power densities for both systems $\frac{I_{D, U W B}}{I_{D, W L A N}}$ for the different persons are now computed using the values of Tables III and V, and are listed in Table VI. On one hand, the LOS component power densities ratio between the UWB and WLAN systems lead to a constant value for all the investigated scenarios, i.e., 0.074, which is also the ratio between the transmitted powers of the UWB $(7.40 \mathrm{~mW})$ and the WLAN $(100 \mathrm{~mW})$ systems. The induced $\mathrm{SAR}_{L O S}$ due to the LOS component is therefore the same for both systems assuming the same transmitted power. On the other hand, the DMC power densities ratios between UWB and WLAN systems vary, and for all the investigated scenarios the ratios are lower than the transmitted

\begin{tabular}{|l|l|l|l|}
\hline Person & $\begin{array}{l}\text { distance } \\
(\mathrm{m})\end{array}$ & $\frac{I_{D, U W B}}{I_{D, W L A N}}$ & $\frac{I_{L, U W B}}{I_{L, W L A N}}$ \\
\hline $\begin{array}{l}\text { p11 } \\
\text { (R1) }\end{array}$ & 3 & 0.0549 & 0.0747 \\
\hline $\begin{array}{l}\text { p12 } \\
\text { (R1) }\end{array}$ & 6 & 0.0404 & 0.0747 \\
\hline $\begin{array}{l}\text { p21 } \\
\text { (R2) }\end{array}$ & 10 & 0.0347 & 0.0770 \\
\hline $\begin{array}{l}\text { p31 } \\
\text { (R3) }\end{array}$ & 8 & 0.0354 & 0.0740 \\
\hline
\end{tabular}

TABLE VI

UWB AND WLAN SYSTEMS POWER DENSITIES RATIO FOR BOTH LOS COMPONENT AND DMC

powers ratio of 0.074 . This points out that for the same transmitted power the exposure to the DMC in an UWB system will be lower than the exposure to the DMC in a WLAN system. Moreover, the equations (15) and (27) confirm that the DMC exposure for UWB systems is always lower than the DMC exposure for the WLAN exposure (for the same transmitted power), regardless of the separation from the Tx. This can also be intuitively explained by the fact that the diffuse energy density evolution never reaches a steady state in the UWB systems - due to the small duration of the pulse unlike the WLAN systems.

For the $\mathrm{SAR}_{w b}$ at the considered frequency, the basic restriction is $\mathrm{SAR}_{w b}=0.08 \mathrm{~W} / \mathrm{kg}$ for the general public exposure and $\mathrm{SAR}_{w b}=0.4 \mathrm{~W} / \mathrm{kg}$ for the occupational exposure [11]. All the values obtained in this paper are below these basic restriction values. Note that the comparison with the basic restrictions values is not the purpose of this paper, it rather aims to determine via an electrical circuit model the actual absorption levels in a realistic environment.

\section{E. Sensitivity of the model to the input parameters}

One could ask whether the computed $\mathrm{SAR}_{w b}$ overestimates or underestimates the actual exposure. Here we determine which of the input parameters are more or less sensitive regarding the $\mathrm{SAR}_{w b}$ estimation. The separation of an exposed person from the $\mathrm{Tx}\left(\mathrm{d}_{0}\right)$ is easily obtained with accuracy. The total absorbing area $A$, the room volume $\mathrm{V}$, and the fraction of the energy being absorbed by the walls $\eta$ are more difficult to obtain, and can therefore be determined with some errors affecting thereby the estimated value of the $\mathrm{SAR}_{w b}$. For all the input parameters ( $\mathrm{V}$ in $\mathrm{m}^{3}, \mathrm{~A}$ in $\left.\mathrm{m}^{2}, \eta\right)$, a variation of $\pm 10 \%$ is assumed. The impact of these variations on the estimated $\mathrm{SAR}_{w b}$ is then investigated. Because there are very low deviations between the theoretical and the simulated $\mathrm{SAR}_{w b}$ values, the $\mathrm{SAR}_{w b}$ values in this part are determined with the theoretical formulas similarly to the Section IV-C1. The $\mathrm{SAR}_{w b}$ values are re-calculated (in $\mu \mathrm{W} / \mathrm{kg}$ ) for the persons $\mathrm{p}_{11}$ and $\mathrm{p}_{12}$ in the room R1 of Fig. 5. $\Delta_{p i j}$ is the relative deviation in $\%$ between the re-calculated value and the actual value of the $\mathrm{SAR}_{w b}$ for the person $\mathrm{p}_{i j}$.

Table VII shows the variation of the $\mathrm{SAR}_{w b}$ values when the input parameters have a variation of $\pm 10 \%$ from their actual values. It turns out that the room volume variation does not affect much the $\mathrm{SAR}_{w b}$ values, the maximum variation being of the order of $\pm 3.7 \%$. An overestimation (resp. underestimation) of the room volume leads to an overestimation (resp. underestimation) of the $\mathrm{SAR}_{w b}$ value. The impact of the area variation on the $\mathrm{SAR}_{w b}$ is much more important than the volume variation impact since the $\mathrm{SAR}_{w b}$ variation may reach $\pm 9 \%$. An overestimation (resp. underestimation) of the 


\begin{tabular}{|c|l|l|l|l|l|l|l|l|}
\hline \multicolumn{2}{|c|}{$\begin{array}{c}\text { input } \\
\text { parameters }\end{array}$} & $\begin{array}{l}\tau \\
(\mathrm{ns})\end{array}$ & $\begin{array}{l}\mathrm{I}_{D, p 11} \\
\left(\mu \mathrm{W} / \mathrm{m}^{2}\right)\end{array}$ & $\begin{array}{l}\mathrm{I}_{D, p 12} \\
\left(\mu \mathrm{W} / \mathrm{m}^{2}\right)\end{array}$ & $\begin{array}{l}\mathrm{SAR}_{w b}^{p 11} \\
(\mu \mathrm{W} / \mathrm{kg})\end{array}$ & $\begin{array}{l}\mathrm{SAR}_{w b}^{p 12} \\
(\mu \mathrm{W} / \mathrm{kg})\end{array}$ & $\begin{array}{l}\Delta_{p 11} \\
(\%)\end{array}$ & $\begin{array}{l}\Delta_{p 12} \\
(\%)\end{array}$ \\
\hline$(\mathrm{V}+10 \%)$ & $330 \mathrm{~m}^{3}$ & 35.27 & 14.4 & 10.9 & 0.24 & 0.096 & 0.8 & 3.3 \\
\hline$(\mathrm{V}-10 \%)$ & $270 \mathrm{~m}^{3}$ & 28.86 & 13.6 & 9.6 & 0.23 & 0.089 & -0.8 & -3.7 \\
\hline$(\mathrm{A}+10 \%)$ & $308 \mathrm{~m}^{2}$ & 29.15 & 12.4 & 8.8 & 0.23 & 0.085 & -3.4 & -8.0 \\
\hline$(\mathrm{A}-10 \%)$ & $252 \mathrm{~m}^{2}$ & 35.63 & 16.1 & 12.1 & 0.245 & 0.101 & 4.5 & 9.8 \\
\hline$(\eta+10 \%)$ & 0.49 & 29.15 & 12.4 & 8.8 & 0.23 & 0.085 & -3.4 & -8.0 \\
\hline$(\eta-10 \%)$ & 0.40 & 35.63 & 16.1 & 12.1 & 0.245 & 0.101 & 4.5 & 9.8 \\
\hline \multicolumn{7}{|c|}{ TABLE VII } \\
\hline
\end{tabular}

IMPACT OF THE INPUT PARAMETERS VARIATION ON THE ACTUAL SAR $w b$ VALUE

absorbing area or of $\eta$ leads to an underestimation (resp. overestimation) of the $\mathrm{SAR}_{w b}$ value. The electromagnetic radiations are absorbed by the surface areas, an underestimation of these surfaces means that the electromagnetic radiation will last longer in the room, and the absorption of an exposed person in terms of $\mathrm{SAR}_{w b}$ will therefore be more important. Similarly, the influence of $\eta$ on the $\mathrm{SAR}_{w b}$ is intuitive. An overestimation of $\eta$ means that the waves are reflected or scattered with less power, the absorption rate of an exposed person to these waves will therefore be lower than the actual value. It is noteworthy to mention that the impact of the input parameters variation depends on the person location as well.

Given that the surface of the possible objects in the room (tables, desk, computers, etc...) have not been accounted in our method, the resulting area is the minimum absorbing area. The $\mathrm{SAR}_{w b}$ value obtained with the present circuit model is therefore the upper limit of the absorption in terms of $\mathrm{SAR}_{w b}$.

\section{CONCLUSions}

A new electrical circuit model for the human exposure and whole-body absorption rate prediction in the indoor environments is proposed. Basically, the method translates the room electromagnetics theory into a electrical circuit, which may be embedded in circuit components. The method accounts for the diffuse multipath component and for the Line-Of-Sight component as well, hence allows the forecast of the whole-body specific absorption rate in a realistic complex environment. Moreover, a simpler method to determine the reverberation time in a realistic environment without the need of carrying out any measurements or simulations is presented. The theory also applies to the adjacent rooms case and an application of the circuit model in a realistic office environment is shown. The strength of the method lies in its simplicity, because it does not require any simulation of the entire room in a software. The theory is applied to an Ultra Wide Band system and to a Wireless Local Area Network system. The lowest absorption rate obtained for the UWB systems is due to the DMC part, whose energy density evolution can not reach the steady state, unlike to the WLAN systems. Future research will consist of the extension of the theory to several coupled rooms.

\section{REFERENCES}

[1] Walter Honcharenko, Henry L. Bertoni, James L. Dailing, J. Qian, and H. D. Yee Mechanisms Governing UHF Propagation on Single Floors in Modern Office Buildings. IEEE Transactions on Vehicular Technology, 41 (4): pages 496-504, 1992.

[2] Majid A. Nemati and Robert A. Scholtz. A Diffusion Model For UWB Indoor Propagation. In Military Communications Conference., 2004.

[3] Majid A. Nemati and Robert A. Scholtz. A Parametric Analytical Diffusion Model for Indoor Ultra-Wideband Received signal. In Conference on Signals, Systems and Computers., 2005.
[4] J. Bach Andersen, J. Ø. Nielsen, G. F. Pedersen, G. Bauch, and M. Herdin. Room Electromagnetics. IEEE Antennas and Propagation Magazine, 49, No. 2: pages 27-33, April 2007.

[5] Nicolai Czink, Andreas Richter, Ernst Bonek, Jukka-Pekka Nuutinen, Juha Ylitalo. Including Diffuse Multipath Parameters in MIMO Channel Models. In IEEE 66th Vehicular Technology Conference, VTC-2007 Fall., 2007.

[6] Juho Poutanen, Jussi Salmi, Katsuyuki Haneda, Veli-Matti Kolmonen, and Pertti Vainikainen. Angular and Shadowing Characteristics of Dense Multipath Components in Indoor Radio Channels. IEEE Transactions on Antennas and Propagation, 59: pages 245-253, 2011.

[7] Jørgen Bach Andersen, Kin Lien Chee, Martin Jacob, Gert Frølund Pedersen, Thomas Kürner. Reverberation and Absorption in an Aircraft Cabin with the Impact of Passengers. IEEE Transactions on Antennas and Propagation, 60 (5): pages 2472-2480, 2012.

[8] Heinrich Kuttruff. Room Acoustics. Spon Press, 2000, London.

[9] Vittorio Degli-Esposti. A Diffuse Scattering Model for Urban Propagation Prediction. IEEE Transactions on Antennas and Propagation, 49 (7): pages 1111$1113,2001$.

[10] A. Bamba, W. Joseph, J. B. Andersen, E. Tanghe, G. Vermeeren, D. Plets, J. O. Nielsen, and L. Martens. Experimental Assessment of Specific Absorption Rate Using Room Electromagnetics. IEEE Transactions on Electromagnetic Compatibility, 54(4): pages 747-757, 2012.

[11] ICNIRP. Guidelines for limiting exposure to time-varying electric, magnetic, and electromagnetic fields (up to $300 \mathrm{GHz}$ ). Health Physics, 74, no. 4: pages 494522, 1998.

[12] Sven Kühn, Wayne Jennings, Andreas Christ and Niels Kuster. Assessment of induced radio-frequency electromagnetic fields in various anatomical human body models. Physics In Medicine And Biology, 54 (2009): pages 875-890, 2009.

[13] T. Kientega. A new approach to assess the specific absorption rate induced by multiple plane waves at $2.1 \mathrm{GHz}$. In EuCAP 2011 - Convened Papers, 2011.

[14] Emmanuelle Conil, Abdelhamid Hadjem, Azeddine Gati, Man-Fai Wong and Joe Wiart. Influence of Plane-Wave Incidence Angle on Whole Body and Local Exposure at $2100 \mathrm{MHz}$. IEEE Transactions on Electromagnetic Compatibility, 53, No. 1: pages 48-52, 2011.

[15] Leen Verloock, Wout Joseph, Günter Vermeeren, and Luc Martens. Procedure for Assessment of General Public Exposure from WLAN in Offices and in Wireless Sensor Metwork Testbed. Health Physics, 98 (4): pages 628-638, 2010.

[16] Ondrej Franek, Jørgen Bach Andersen and Gert Frølund Pedersen. Diffuse Scattering Model of Indoor Wideband Propagation. IEEE Transactions on Antennas and Propagation, 59, No. 8: pages 3006-3012, 2011.

[17] Bruce Hapke. Theory of Reflectance and Emittance Spectroscopy. Press Syndicate of the University of Cambridge, 1993.

[18] Francesco Mani, Francois Quitin, and Claude Oestges. Accuracy of depolarization and delay spread predictions using advanced ray-based modeling in indoor scenarios. EURASIP Journal on Wireless Communications and Networking. Available online: http://jwcn.eurasipjournals.com/content/2011/1/11, no. 11:11 pages, 2011 .

[19] Andreas F. Molisch. Ultrawideband Propagation Channels-Theory, Measurement, and Modeling. IEEE Transactions on Vehicular Technology, 54, No. 5: pages $1528-1545,2005$.

[20] Agilent Technologies. Advanced design system, ads. http://www.home.agilent.com.

[21] A. Bamba, W. Joseph, G. Vermeeren, E. Tanghe, D. P. Gaillot, J. B. Andersen, J. $\varnothing$. Nielsen, M. Lienard and L. Martens. Validation of Experimental whole-body SAR Assessment Method in a Complex Environment. Bioelectromagnetics, 34 (2): pages 122-132, 2013. 